\title{
New design of dual-band bandpass microwave filter based on electromagnetic effect of metamaterial resonators
}

\author{
Mohammed Berka $^{* * *}$, Zoubir Mahdjoub ${ }^{* *}$, Mourad Hebali $^{* * *}$
}

\begin{abstract}
Recent research on microwave filters show that there are constraints in their design; Effective solutions are needed for highquality systems. In this manuscript, a new design of microwave dual-band bandpass filter is presented. The complementary metamaterial resonators with magnetic activity and negative permeability $(\mu<0)$ are engraved on the lower ground plane and coplanar guides are maintained in the upper plane of the filter. Both (CSRRs) used have different shapes and dimensions from one another to have a dual band-pass behavior of our filter outside the range 5 to $6 \mathrm{GHz}$ to avoid clutter with other microwave transmission systems. The supply lines used for the inlet and the filter outlet has coplanar access. To show the influence of the coupling of the two metamaterials resonators with the coplanar waveguides on the qualities of the filter, we do simulations using HFSS commercial software.
\end{abstract}

K e y w o r d s: CSRRs, dual-band (BPF), metamaterial, permeability, resonator

\section{Introduction}

Currently, one of the most desired objectives by the wireless communications industry is to improve the electrical performance of microwave filters, including bandpass filters that this type of filter is frequently used in modern telecommunication devices. Recent work on this kind of filter show a great development in the microwave filtering qualities, such as the miniaturization of the circuits [1], the selectivity [2] and tunability [3] of the filters. However, the major problem that can disrupt these qualities is the intersystem congestion [4].

In the field RF/Microwave, the choice of materials is of great importance for the realization of filters. A study of the physical properties of these materials is therefore essential to improve the performance of such a filter. Now, a new class of physical devices can meet the needs of the performance of microwave systems, these devices are named "Metamaterials".

Metamaterials are pseudo-homogeneous structure having new physical characteristics. The theoretical study of the main properties of metamaterials was made by Victor Veselago [5], he has showed the advantages of this media. The electromagnetic properties of metamaterials concern the permeability $(\mu)$, the permittivity $(\varepsilon),[6]$ and the refractive index $(n),[7]$. The geometric properties concern the dimensions of the resonators $[8,9]$ toward wavelength within the structures. In 1999, Sir John Pendry introduced a new structure called the "swiss-roll", this structure has anisotropic behavior [10] undesirable because of its remarkable absorption. To reduce this absorption, Pendry proposed another structure based on split rings, often called "Splits Rings Resonators" or (SRRs). Subsequently and according to the desired objectives of these resonators, several geometric shapes are displayed as $\ll U, \Omega \gg$ shapes [11,12] and the triangular form [13].

The aim of this manuscript is to have a new design of microwave dual-band bandpass filter. For this reason, we used the defected ground structure technology (DGS) [14] for a coplanar waveguide which has lower ground plane, we used two resonators of different shapes and sizes and at the same time, we look for the proper position of these (CSRRs) in the ground plane to have the necessary coupling that meets the requirements of our global filter.

\section{Design methodology}

\subsection{Characteristics of the used substrate}

In microwave, the physical properties of the components constituting any circuit perform an important role in the analysis and synthesis of this circuit. In planar or coplanar technology, the physical characteristics of the substrate have a great influence on the qualities of the microwave devices. Therefore, a good choice of the material constituting the substrate is required to improve the qualities of the planar and/or coplanar circuits. Electromagnetic materials are defined by their relative permittivity $\left(\varepsilon_{r}\right)$ and permeability $\left(\mu_{r}\right)$ related as follows

$$
\left\{\begin{array}{l}
\varepsilon_{r}=\varepsilon_{r}^{\prime}-j \varepsilon_{r}^{\prime \prime} \\
\mu_{r}=\mu_{r}^{\prime}-j \mu_{r}^{\prime \prime}
\end{array}\right.
$$

Or by their permittivity $(\varepsilon)$ and permeability $(\mu)$ using the following expressions

$$
\left\{\begin{array}{l}
\varepsilon=\varepsilon_{0} \varepsilon_{r}^{\prime}\left(1-j \tan \left(\delta_{\varepsilon}\right)\right. \\
\mu=\mu_{0} \mu_{r}^{\prime}\left(1-j \tan \left(\delta_{\mu}\right)\right.
\end{array}\right.
$$

* Department of Electrical engineering, University of Mascara, Algeria, ** EPO Laboratory, University of Sidi -B-Abbess, Algeria, *** Department of Electrical Engineering, ENP Oran, Laboratory CaSiCCE, 31000 Oran, Algeria, mohammed_76_berka@yahoo.fr 


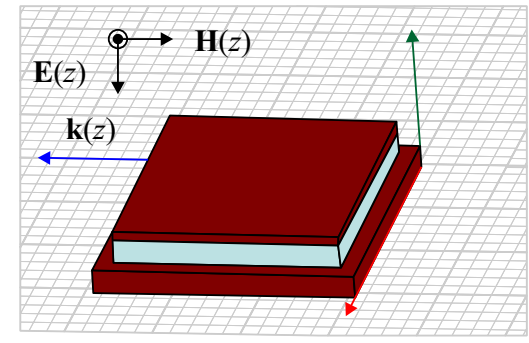

(a)

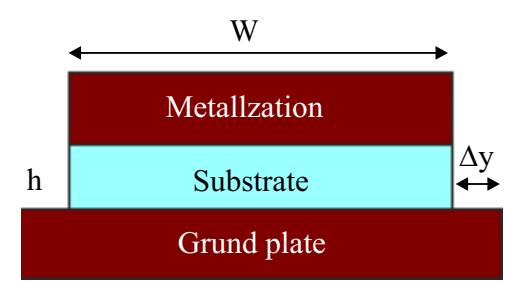

(b)

Fig. 1. Cavity filled with three dielectrics: (a) - 3-D model on HFSS, (b) - cross section

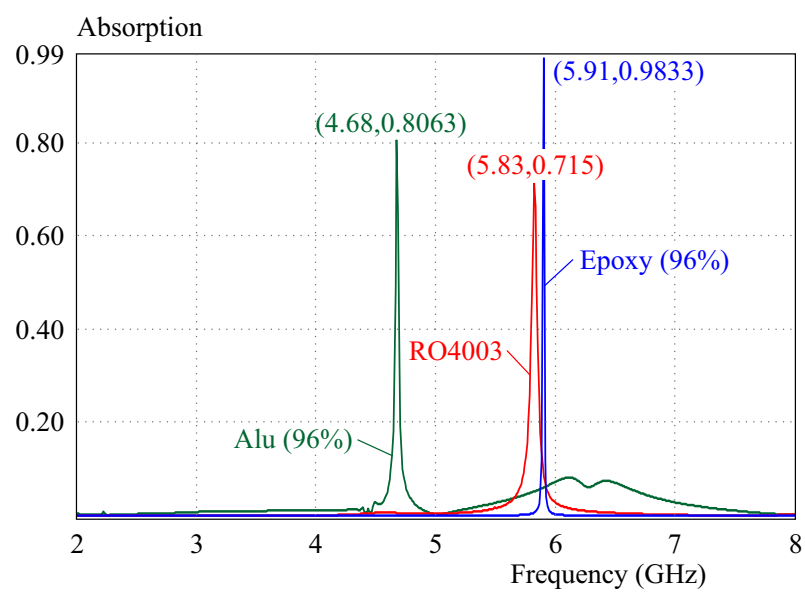

Fig. 2. Absorption characteristics of the three selected materials

whose loss characteristics (dielectric loss tangent) are given by

$$
\left\{\begin{array}{l}
\delta_{\varepsilon}=\tan ^{-1}\left(\frac{\varepsilon_{r}^{\prime \prime}}{\varepsilon_{r}^{\prime}}\right) \\
\delta_{\mu}=\tan ^{-1}\left(\frac{\mu_{r}^{\prime \prime}}{\mu_{r}^{\prime}}\right)
\end{array}\right.
$$

where $\varepsilon_{0}=8.85 \times 10^{-12} \mathrm{~F} / \mathrm{m}$, and $\mu_{0}=4 \pi \times 10^{-7} \mathrm{H} / \mathrm{m}$ are the vacuum permittivity and permeability. To perform the selection on the substrate, we will compare the absorption of three materials (often used for planar and/or coplanar circuits) of the same thickness $h$ from three square shaped Cavity fulfilled by three materials $\left(\mu_{r}=1\right)$ located between two conductive planes (Copper). The absorption in each structure is given by the following relationship, with $S_{21}=0,[15]$

$$
A_{b}=1-\left|S_{11}\right|^{2}
$$

The thicknesses of the two planes drivers do not have importance. We polarize our structure by a plane wave in the electric field is along the axis $O_{z}$, HFSS simulator gives the absorption characteristics of the three materials as follows from Fig. 2.

Table 1 with $W=2.6 \mathrm{~mm}, h=0.35 \mathrm{~mm}$ and $\Delta y=$ $0.2 \mathrm{~mm}$ for each material, summarizes our results with the dimensions of the proposed structure.
Table 1.

\begin{tabular}{lcccc}
\hline Materials & $\varepsilon_{r}^{\prime \prime}$ & $\begin{array}{c}A_{b} \\
(\%)\end{array}$ & $\begin{array}{c}f_{r} \\
(\mathrm{GHz})\end{array}$ & $h_{\lambda}$ \\
\hline Epoxy FR4 & 0.088 & 98.33 & 5.91 & $\lambda / 145$ \\
Alumina 96\% & 0.056 & 80.63 & 4.68 & $\lambda / 183$ \\
Rogers (4003) & 0.009 & 71.50 & 5.83 & $\lambda / 147$ \\
\hline
\end{tabular}

We chose the portions $(W, h, \Delta y)$ to get the absorption in the band rejection, allowing us to choose the best substrate in the design of our filter. According to our results, we note that the substrate Rogers (4003) is the most suitable with less absorption and a thickness of about $(\lambda / 147)$ around the resonant frequency $\left(f_{r}=5.83 \mathrm{GHz}\right)$.

\section{2 Properties of used complementary metamaterial resonators}

The electromagnetic characteristics of complementary split ring resonator (CSRRs) are different from those of the metamaterial resonator (SRRs). For the geometric characteristics, the (CSRRs) also has very small dimensions to the wavelength at resonance. In physical terms, the (CSRRs) is the complement of (SRRs), ie everything that is in the conductive tracks in (SRRs) becomes slots in the (CSRRs) and vice versa. The (SRRs) work correctly when the magnetic field is perpendicular to the rings, so the (CSRRs) resonate when the electric field is perpendicular to the same rings. Both (CSRRs) proposed in this work have two different forms, the first Complementary resonator in a spiral shape and the second is circular but in symmetrical type (CSRRSs) (complementary split ring resonators symmetrical).

The (CSRRs) spiral is formed by two circular rings connected by an inclined slot. For (CSRRSs) there are two inner and outer rings, each one has two interruptions of the slot. In the outer ring, the two interruptions are in the $\boldsymbol{H}$ direction, while for the inner ring the interruptions are in the $\boldsymbol{k}$ direction. We represent the two (SRRs) in different shape and size and his complementary resonators (CSRRs) in Fig. 3 and Fig. 4.

The (CSRRs) spiral can be regarded as mainly an electric dipole, its equivalent circuit model behaves like a $L_{0} C_{0}$ resonator excited by an electric field perpendicular to the plane of the rings [16]. For the equivalent 


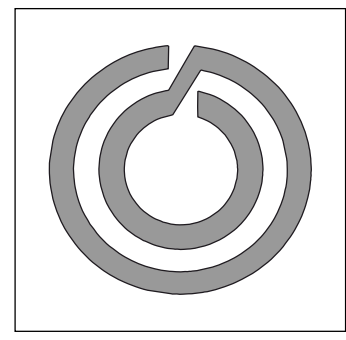

(a)

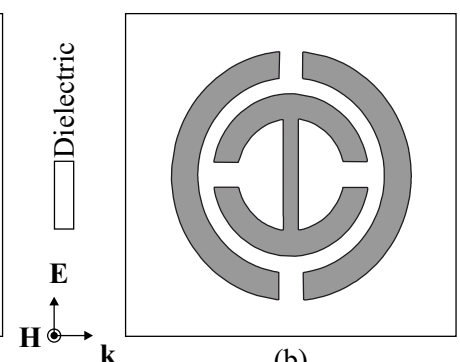

(b)

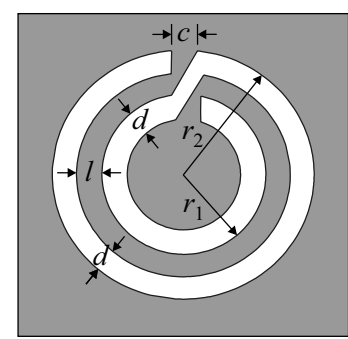

(a)

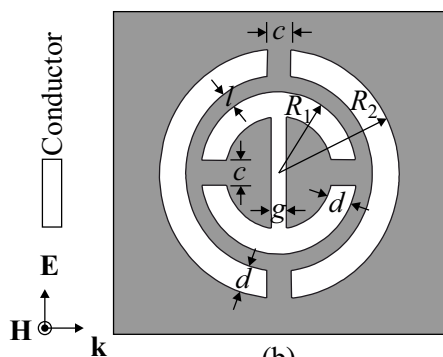

(b)

Fig. 3. SRRs shapes: (a) - spiral, (b) - symmetrical circular

Fig. 4. CSRRs shapes: (a) - spiral, (b) - symmetrical circular

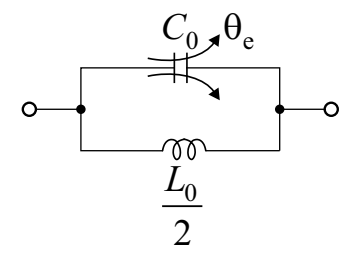

(a)

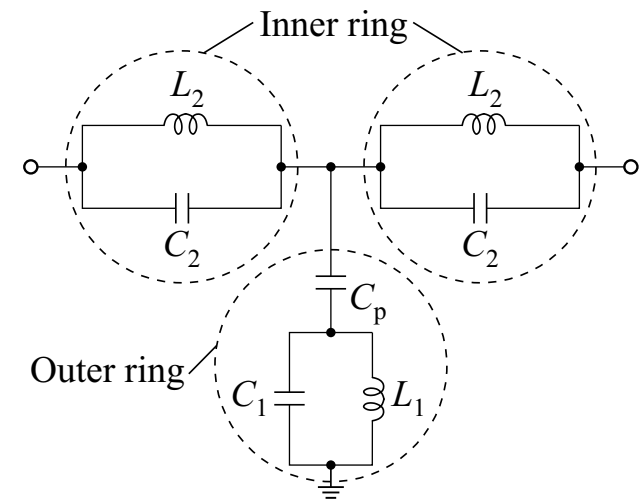

(b)

Fig. 5. Equivalent circuit model for: (a) - (CSRRs) spiral, (b) - (CSSRRs)

circuit (CSSRRs) there are two series resonators compounds of the inductance $L_{1}$ and the capacitance $C_{1}$, these resonators represent the two defected half-circles to the outer ring. The inner ring with the additional connection slot corresponds to the resonator formed by the inductance $L_{2}$ and capacity $C_{2}$. The $C_{p}$ capacity represents the coupling between the inner and outer rings [17]. Figure 5. shows the two equivalent circuit models.

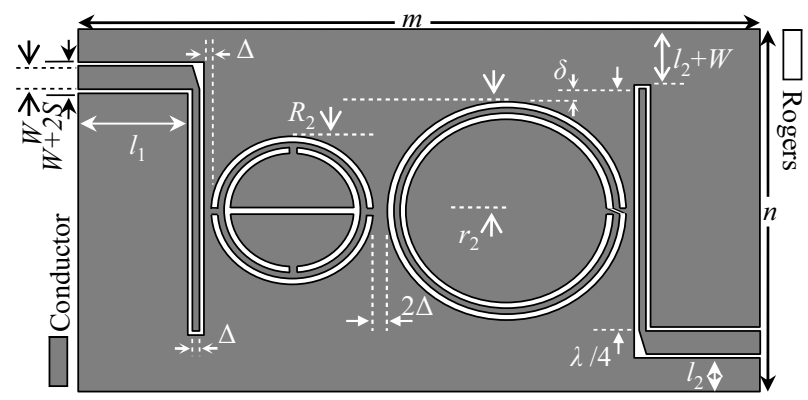

Fig. 6. Top view of the global filter

\subsection{Proposed dual-band (BPF)}

The overall filter consists of two coplanar lines coupled in parallel in the upper surface of (RO4003) substrate. For these coupled lines, the electromagnetic coupling between the two lines depends on the technological limit; it will be a maximum coupling when the two lines are close to each other (spacing of about $20 \mu \mathrm{m})$. The (CSRRs) are located in the area between the two coplanar lines but the etching is done in the bottom ground plane of the substrate. When the two (CSRRs) for different shapes and sizes are located between the coplanar lines, the spacing distance is important which causes a high level of losses and a much narrower bandwidth.

Our design is to improve the level of coupling with the (CSRRs) without increasing the level of losses. A distance $(\Delta+S)$ between each coplanar line and one of two (CSRRs) is fixed when the $(\Delta)$ value takes the half of the thickness of the substrate used, the width of each line is the same value as $(\Delta)$. The coplanar lines are quarterwave $(\lambda / 4)$ to the magnetic resonance of the smaller (SRRs), they have the same length to have a symmetrical coupling. The dimensions of the two (CSRRs) are selected to have resonances of (SRRs) outside the range 5-6 GHz. The (CSRRs) are placed in the lower face of the substrate such that their interruptions are oriented to the coplanar lines from one another to increase the level of coupling. The spacing between the two (CSRRs) is the order of $(2 \Delta)$. The overall filter is shown in Fig. 6 . 


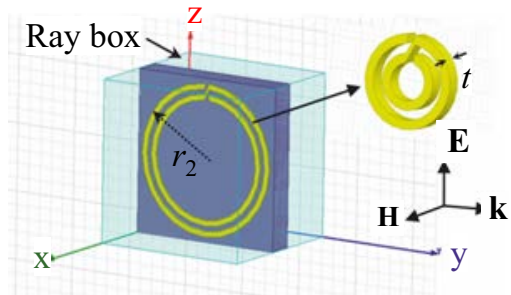

(a)

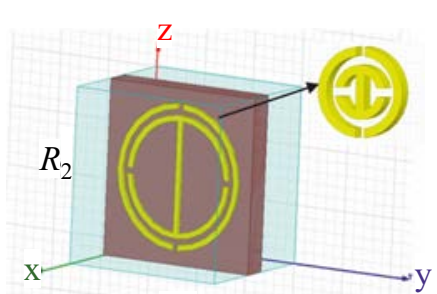

(b)

Fig. 7. 3-D view: (a) - spiral (SRRs), (b) - symmetrical circular (SRRs)

Table 2. Electromagnetic characteristics of both SRRs

\begin{tabular}{lcccccc}
\hline & \multicolumn{3}{c}{$R_{2}(\mathrm{~mm})$} & \multicolumn{3}{c}{$r_{2}(\mathrm{~mm})$} \\
& 1.5 & 1.7 & 1.9 & 2.8 & 3.2 & 3.4 \\
\hline$f_{r}(\mathrm{GHz})$ & 6.80 & 6.70 & 6.43 & 4.57 & 4.38 & 4.17 \\
$* P_{R}(\mathrm{~dB})$ & -11.93 & -21.6 & -21.8 & -27.5 & -17.9 & -21.4 \\
\hline$*$ Return
\end{tabular}

Table 3. Overall filter Dimensions in mm

\begin{tabular}{cccc}
\hline$R_{2}$ & $r_{2}$ & $\lambda / 4$ & $\delta$ \\
1.9 & 2.8 & 6.2 & 0.3 \\
\hline$W$ & $\Delta$ & $l_{1}$ & $l_{2}$ \\
0.6 & 0.175 & 2.575 & 0.85 \\
\hline
\end{tabular}

\section{Discussion of results}

\subsection{Electromagnetic characteristics of(srrs)}

The electromagnetic characteristics of (SRRs) depend essentially on the dimensions of these complementary resonators). Here, the choice of the diameter plays the major role to obtain the different resonances. The two resonators are shown in Fig. 7.

On the simulator, the polarization of the two structures is in the $O_{z}$ direction. We define both electric and magnetic wall, the thickness $(t)$ of each (SRRs) on copper is of the order of $5 \mu \mathrm{m}$. We stimulate our resonators for three different values of $r_{2}$ and $R_{2}$. The dimensions ( $c=d=l=0.15 \mathrm{~mm}$ ) are identical in the two forms of resonators and $(g=0.15 \mathrm{~mm})$ in the symmetrical circular resonator. The (SSRs) spiral behavior can be represented by Fig. 8. and that of the (SRRs) symmetrical circular is shown in Fig. 9.

The characteristics of the two shapes of (SRRs) are summarized in Table 2.

From Table 2, we can choose the two complementary resonators according to the geometric and electromagnetic characteristics of the split resonators (SRRs) for the two proposed forms. To miniaturize the global filter and to be able to contain both (CSRRs) between the two coplanar guides (CPW), we chose the spiral (CSRRs) which has the dimensions $(c=d=l=0.15 \mathrm{~mm}$ and $r_{2}=2.8 \mathrm{~mm}$ ) and that of the symmetrical circular shape which has the dimensions $(c=d=l=0.15 \mathrm{~mm}$ and $\left.R_{2}=1.9 \mathrm{~mm}\right)$.

\subsection{Overall filter response}

According to our previous results on the characteristics of (SRRs) and the physical and geometrical characteristics of the substrate used, we can characterize our filter in Table 3.

Our filter has length and width $(m=16 \mathrm{~mm}, n=$ $9.3 \mathrm{~mm}$ ), on the 3-Modeler of HFSS simulator, our filter can be represented in Fig. 10.

The filter response is illustrated in Fig. 11.

The phase parameters of the overall filter are shown in Fig. 12.

Figure 11 shows in the frequency range 2 to $10 \mathrm{GHz}$ the response of our overall structure, it is noted that the latter has a filtering behavior. It is indeed a dual-band bandpass filter. It may be noted that both of our filter bandwidths are outside the 5 to $6 \mathrm{GHz}$ band, which justifies our choice on complementary metamaterial resonators (CSRRs) on both counts; shapes and dimensions. We can also say that the resonances of our filter are remoted to the band 5 to $6 \mathrm{GHz}$, especially to low frequency ( $5 \mathrm{GHz}$ ). On the same response from our filter, the two zero frequencies can be $\operatorname{noted}\left(f_{\mathrm{z} 1}=3.06 \mathrm{GHz}, f_{\mathrm{z} 2}=8.46 \mathrm{GHz}\right)$.

\section{Conclusion}

A new dual-band bandpass filter is presented in this paper; the characteristics of the filter are obtained by the coupling of the coplanar waveguide (CPW) with complementary metamaterial resonators. In our design we used two different (CSRRs) maintained in the lower ground plane to ensure a sufficient coupling with the CPW despite for a considerable distance and also to have (SRRs) magnetic resonance outside the 5 to $6 \mathrm{GHz}$ band. Both (CSRRs) has allowed us to reinforce this coupling with the least possible losses which gives us a remarkable robustness to our overall structure, this structure has a rejection in the 5 to $6 \mathrm{GHz}$ band. So, we obtained the desired electrical qualities for our filter. 


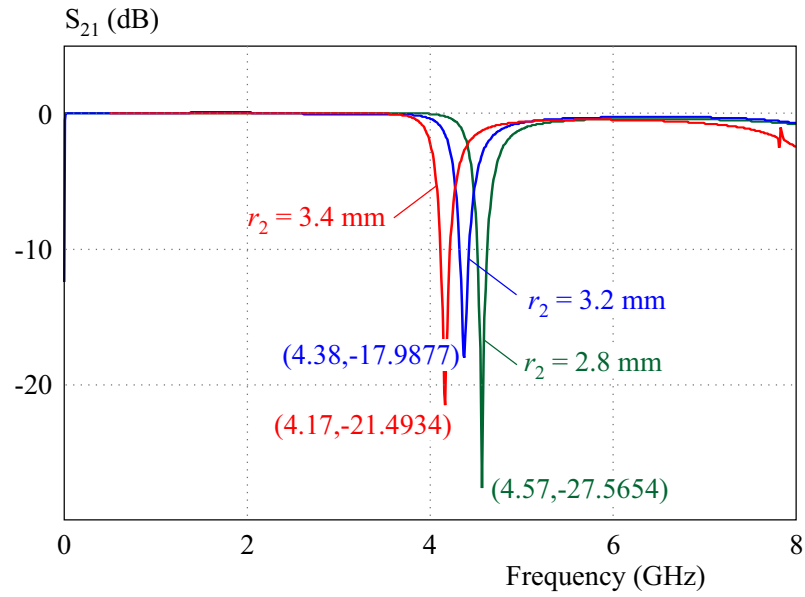

Fig. 8. Transmission of spiral (SRRs) for the three rays

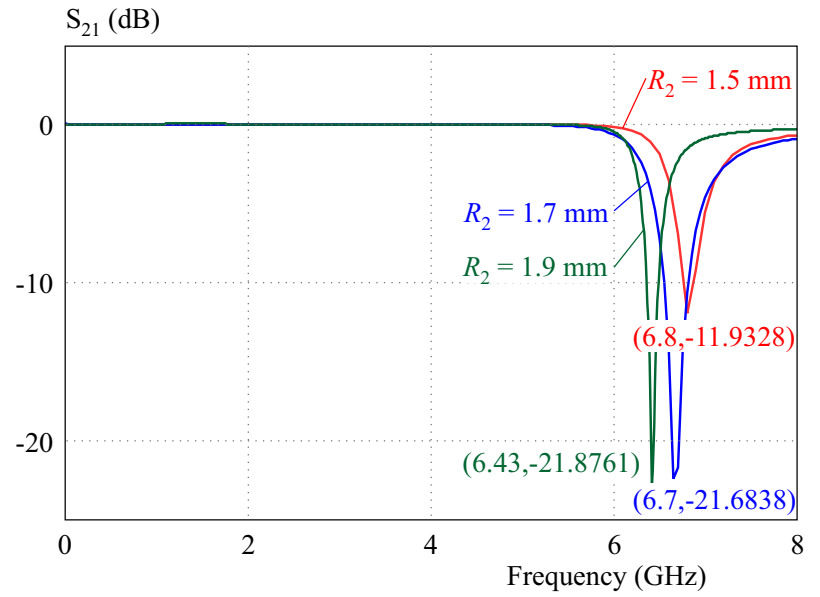

Fig. 9. Transmission of symmetrical circular (SRRs) for the three rays

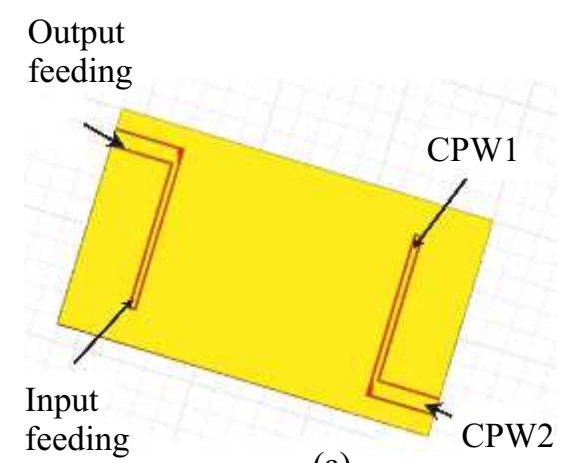

(a)

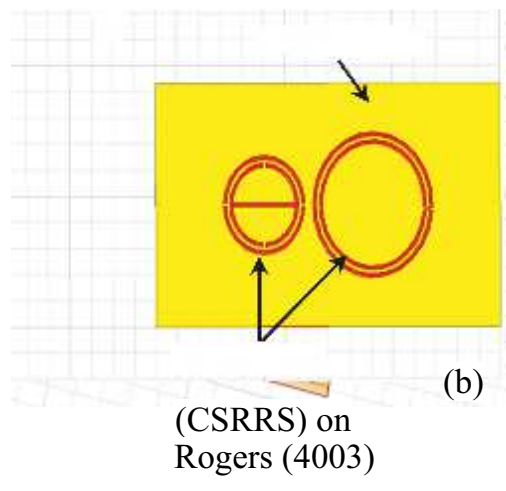

(b)
Rogers (4003)

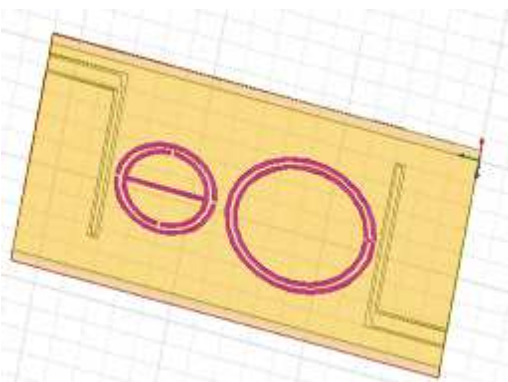

(c)

Fig. 10. Overall filter: (a) - top view, (b) - bottom view, (c) - 3-D view

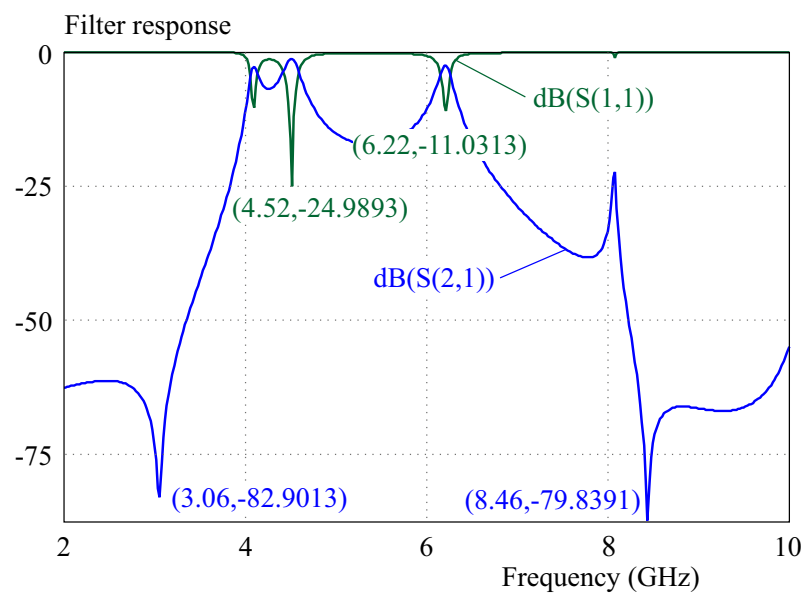

Fig. 11. Overall filter response

\section{REFERENCES}

[1] G. A. Kouzaev, "Integrated Filters and Power Distribution Circuits", Applications of Advanced Electromagnetic vol. 169, 2013, pp. 341-357.

[2] J. Xu, Y. Ji, C. Miao and W. Wu, "Compact Bandpass Filter with High Selectivity and Wide Stopband using Slotted Stepped-Impedance Resonator", Journal of Electronics vol. 29, 2012, pp. 23-26.

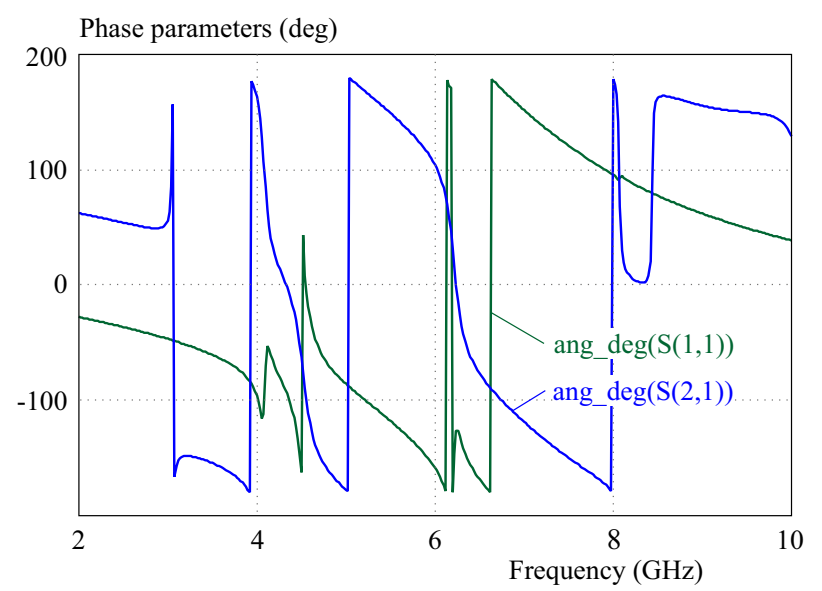

Fig. 12. Phase parameters of overall filter

[3] N. Benmostefa, M. Meliani and H. Ouslimani, "Metamaterial Tunable Filter Design", Journal of Electromagnetic Analysis and Applications vol. 5, 2013, pp. 250-254.

[4] Yuan Gao, Yuanjin Zheng and Ban-Leong Ooi, "A 0.18-/spl $\mathrm{mu} / \mathrm{m}$ CMOS UWB LNA with 5GHz Interference Rejection", IEEE Radio Frequency Integrated Circuits (RFIC) Symposium, 2007, pp. 47-50. 
[5] V. G. Veselago, "The Electrodynamics of Substances with Simultaneously Negative Values of $\varepsilon$ and $\mu$ ", Soviet Physics Uspekhi vol. 10, 1968, pp. 509-514.

[6] R. Merlin et al, "Metamaterials and the Landau-Lifshitz Permeability Argument, Large Permittivity Begets High-Frequency Magnetism", National Academic Science vol. 10, 2009, pp. 1693-1698.

[7] N. Estep, A. Askarpour and S. Shvets, "Transmission-Line Model and Propagation a Negative-Index, Parallel-Plate Metamaterial to Boost Electron-Beam Interaction", Antenna and propagation, IEEE Transactions vol. 62, 2014, pp. 3212-3221.

[8] N. Liu, H. Guo, L. Fu and S. Kaiser, "Three Dimensional Photonic Metamaterials at Optical Frequencies", Nature Mater vol. 7, 2008, pp. 31-37.

[9] N. Asfahani and N. Schunemann, "Miniaturized Surface wave Cylindrical Metamaterial Loaded Resonator", Microwave Conference (GeMic), 2012.

[10] R. Marquez and F. Medina, "Role of Bianisotropy Negative Permeability and Left-Handed Metamaterials", Physical Review $B$ vol. 65, no. 14, 2002, pp. 14440-14444.

[11] A. Yang, Y. Chang-Chan, J. Tian and D. Zhang, "A Sensitive Sensor with a Double U-shaped Rings-based Metamaterial", META' 14 - the $5^{\text {th }}$ International Conference on Metamaterials, Photonic Crystals and Plasmonics, Singapore, 2014.

[12] A. Quarchi and H. Belkebir, "Extraction of Effective Parameters of Double Omega Shaped Metamaterial", J. Mater Environ Science vol. 2, 2011, pp. 07-12.

[13] C. Sabah et al, "Tunable Metamaterial Design Composed of Triangular (SRR) and Wire Strip for S and C Microwave Bands", Progress Electromagnetic Researches, 2010.

[14] A. Kumar and M. Kartikeyan, "A Design of Microstrip Bandpass Filter with Narrow Bandwidth using DGS/DMS for WLAN", Communications (NCC), National Conference, 2013.

[15] A. Sellier, T. Teperik, S. Burokur and A. de Lustrac, "Design and Model of Wide Band Absorber Made of Ultrathin Metamaterial Structures", Applied Physics A vol. 117, 2014, pp. 739-746.
[16] F. Falcon, T. Lopetegi, J. D. Banea and R. Marquez, "Babinet Principle Applied to the Design of Metasurfaces and Metamaterials", Phys. Rev. Lett. vol. 93, 2004, pp. 197401-197404.

[17] Q. Xiao, L. X. Wang and X. W. Shi, "A Novel Symmetrical Split Ring Resonator (SRR) based on DGS Technology Supports the Design of Lowpass Filters Wide Rejection Bands", Microwave and RF, 2009.

Received 10 July 2018

Mohammed Berka, Laboratory EPO in SBA, 22000 Algeria. Was born in Algeria in 1976, he received the PhD degree in Telecommunication systems from the SBA university, Algeria in 2015, he joined EPO as a searcher, in 2008. Currently, he is a professor at the University of Mascara. His research interests include microwave devices, antennas, filters, radar, and currently he directs his research towards the design of microwave devices based on metamaterials.

Zoubir Mahdjoub, Laboratory EPO in SBA, 22000 Algeria. Was born in Algeria in 1956, he received the $\mathrm{PhD}$ degree in Electronic from the Grenoble university, France in 1987, he joined EPO as a searcher, in 2008. He is a professor at the University of SBA, he is responsible for several research projects. His research interests include microwave devices, antennas, filters and radar.

Mourad Hebali, Laboratory CaSiCCE, ENPO in Oran, BP1523 Oran Algeria. Was born in Algeria in 1987, he received the MS degree in electronics from the ENP Oran, Algeria in 2014, he joined CaSiCCE laboratory as a PhD student in 2015 and received the $\mathrm{PhD}$ degree in Electrical Engineering of ENP Oran-MA Algeria in 2018. His research interests include semiconductor physics, BSIM3v3 of temperature sensors, ISFET transistor for $\mathrm{pH}$ sensors, HEMT, SOI, DG-MOSFET, design and simulation of micro and nanoelectronics components and circuits. 